\title{
Evaluating the Impact of a Novel Warning Message Dissemination Scheme for VANETs Using Real City Maps
}

\author{
Francisco J. Martinez ${ }^{1}$, Manuel Fogue ${ }^{1}$, Manuel Coll ${ }^{1}$, Juan-Carlos Cano ${ }^{2}$, \\ Carlos T. Calafate ${ }^{2}$, and Pietro Manzoni ${ }^{2}$ \\ 1 University of Zaragoza, Spain \\ \{f.martinez,m.fogue,m.coll\}@unizar.es \\ 2 Universidad Politécnica de Valencia, Spain \\ \{jucano, calafate,pmanzoni\}@disca.upv.es
}

\begin{abstract}
In traffic safety applications for Vehicular ad hoc networks (VANETs), warning messages have to be disseminated in order to increase the number of vehicles receiving the traffic warning information. Hence, redundancy, contention, and packet collisions due to simultaneous forwarding (usually known as the broadcast storm problem) are prone to occur. In the past, several approaches have been proposed to solve the broadcast storm problem in multi-hop wireless networks such as Mobile ad hoc Networks (MANETs). Among them we can find counter-based, distance-based, location-based, cluster-based, and probabilistic schemes. In this paper, we present the enhanced Street Broadcast Reduction (eSBR), a novel scheme for VANETs designed to mitigate the broadcast storm problem in real urban scenarios. We evaluate the impact that our scheme has on performance when applied to VANET scenarios based on real city maps.
\end{abstract}

Keywords: VANETs, real city maps, message dissemination.

\section{Introduction}

Vehicular ad hoc networks (VANETs) are wireless communication networks that do not require any sort of fixed infrastructures, offering a novel networking paradigm to support cooperative driving applications on the road. VANETs are characterized by: (a) constrained but highly variable network topology, (b) specific speed patterns, (c) time and space varying communication conditions (e.g., signal transmissions can be blocked by buildings), (d) road-constrained mobility patterns, and (e) no significant power constraints.

VANETs have many possible applications, ranging from inter-vehicle communication and file sharing, to obtaining real-time traffic information (such as jams and blocked streets), etc. In this work we focus on traffic safety and efficient warning message dissemination, where the objective is to reduce the latency and to increase the accuracy of the information received by nearby vehicles when there is a dangerous situation. 
In urban vehicular wireless environments, an accident can cause many vehicles to send warning messages, and all vehicles within the transmission range will receive the broadcast transmissions and rebroadcast these messages. Hence, a broadcast storm (serious redundancy, contention and massive packet collisions due to simultaneous forwarding) will occur and must be reduced [1]. In the past, several schemes have been proposed to reduce the broadcast storm problem. However, they have been only validated using simple scenarios such as a highway (several lanes, without junctions) [23, or a Manhattan-style grid scenario [4].

In this work, we propose a novel scheme called enhanced Street Broadcast Reduction (eSBR), which uses location and street map information to facilitate the dissemination of warning messages in 802.11p based VANETs. We evaluate the performance of our eSBR algorithm in a realistic urban scenario, that is, with a complex set of streets and junctions, and demonstrate how it could improve performance.

This paper is organized as follows: Section 2 reviews the related work on the broadcast storm problem in wireless ad hoc networks. Section 3 presents a brief description of the eSBR scheme and how it works in a real map scenario. Section 4 presents the simulation environment. Simulation results are then discussed in Section 5. Finally, Section 6 concludes this paper.

\section{Related Work}

In VANETs, intermediate vehicles act as message relays to support end-toend vehicular communications. For applications such as route planning, traffic congestion control, and traffic safety, the flooding of broadcast messages commonly occurs. However, flooding can result in many redundant rebroadcasts, heavy channel contention, and long-lasting message collisions 11. Over the years, several schemes have been proposed to address the broadcast storm problem in wireless networks. They are:

1. The Counter-based scheme 1]. To mitigate broadcast storms, this scheme uses a threshold $C$ and a counter $c$ to keep track of the number of times the broadcast message is received. Whenever $c \geq C$, rebroadcast is inhibited.

2. The Distance-based scheme [1]. In this scheme, authors use the relative distance $d$ between vehicles to decide whether to rebroadcast or not. It is demonstrated that when the distance $d$ between two vehicles is short, the additional coverage (AC) of the new rebroadcast is lower, and so rebroadcasting the warning message is not recommended. If $d$ is larger, the additional coverage will also be larger.

3. The Location-based scheme presented in [1] is very similar to the distancebased scheme, though requiring more precise locations for the broadcasting vehicles to achieve an accurate geometrical estimation (with convex polygons) of the AC of a warning message. Since vehicles usually have GPS systems on-board, it is possible to estimate the additional coverage more precisely. The main drawback for using this scheme is the high computational cost of 
calculating the $\mathrm{AC}$, which is related to calculating many intersection areas among several circles.

4. The weighted p-persistence, the slotted 1-persistence, and the slotted p-persistence techniques presented in [5] are some of the few rebroadcast schemes proposed for VANETs. These three probabilistic and timer-based broadcast suppression techniques are not designed to solve the broadcast storm problem, but they can mitigate the severity of the storm by allowing nodes with higher priority to access the channel as quickly as possible. These schemes are specifically designed for use in highway scenarios.

5. The Last One (TLO) scheme, presented in [2], tries to reduce the broadcast storm problem finding the most distant vehicle from the warning message sender, so this vehicle will be the only allowed to retransmit the message. This method uses GPS information from the sender vehicle and the possible receivers to calculate the distance. Although it brings a better performance than simple broadcast, this scheme is only effective in a highway scenario because it does not take into account the effect of obstacles (e.g. buildings) in urban radio signal propagation. Moreover, GPS information must be accurate to achieve good results, and it is not clearly stated how a node knows the position of nearby vehicles at any given time.

6. The TLO approach was extended using a protocol which utilizes adaptive wait-windows and adaptive probability to transmit, named Adaptive Probability Alert Protocol (APAL) 3. This scheme shows even better performance than the TLO scheme, but it is also only validated in highway scenarios.

Note that all these existing schemes alleviate the broadcast storm problem by inhibiting certain vehicles from rebroadcasting, reducing message redundancy, channel contention, and message collisions. In particular, they inhibit vehicles from rebroadcasting when the additional coverage (AC) area is very low. In [1, the authors demonstrated that a rebroadcast can only provide up to $61 \%$ additional coverage over that area already covered by the previous transmission in the best case (on average, the additional area is of $41 \%$ ).

\section{The Enhanced Street Broadcast Reduction (eSBR) Scheme in Real Maps}

In this section, we present the enhanced Street Broadcast Reduction scheme (eSBR) - our novel proposal to reduce the broadcast storm problem in real urban scenarios. In urban scenarios, and at the frequency of $5.9 \mathrm{GHz}$ (i.e., the frequency band adopted by the 802.11 p standard), radio signals are highly directional and will experience a very low depth of penetration. Hence, in most cases, buildings will absorb radio waves at this frequency, making communication only possible when the vehicles are in line-of-sight.

In our simulations, vehicles operate in two modes: (a) warning, and (b) normal. Warning mode vehicles inform other vehicles about their status by sending warning messages periodically (every $T_{w}$ seconds). These messages have the 


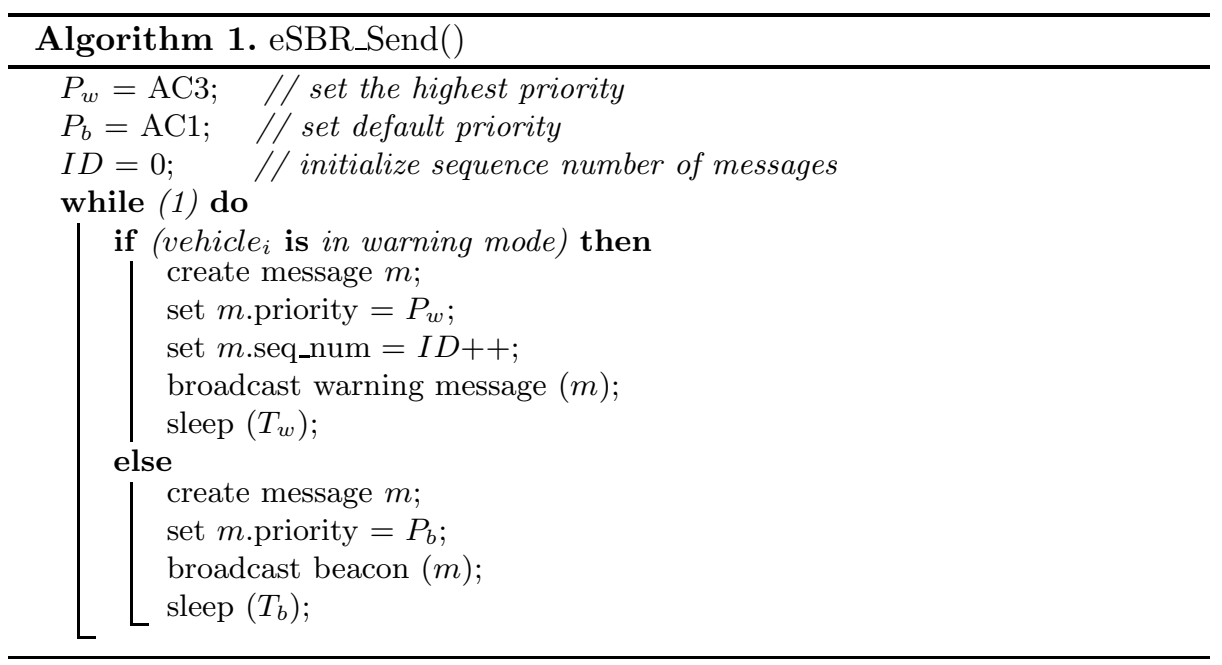

highest priority at the MAC layer. Normal mode vehicles enable the diffusion of these warning packets and, periodically (every $T_{b}$ seconds), they also send beacons with information such as their positions, speed, etc. These periodic messages have lower priority than warning messages and are not propagated by other vehicles. With respect to warning messages, each vehicle is only allowed to propagate them once for each sequence number, i.e., older messages are dropped.

Algorithms 1 and 2 describe our eSBR scheme, where vehicle indicates each vehicle in the scenario; $m$ indicates each message sent or received by each vehicle; warning represents a warning message generated by a warning mode vehicle; beacon represents a normal message generated by an normal vehicle; $T_{w}$ is the interval between two consecutive warning messages; $T_{b}$ is the interval between two consecutive normal messages; $P_{w}$ indicates the priority of the warning messages and $P_{b}$ indicates the priority of the normal messages.

When vehicle $_{i}$ starts the broadcast of a message, it sends $m$ to all its neighbors. When another vehicle receives $m$ for the first time, it rebroadcasts it by further relaying $m$ to its neighbors. Depending on their characteristics, every vehicle repeats send(warning) or send(beacon) operations periodically with different periods ( $T_{w}$ and $T_{b}$, respectively). When a new message $m$ is received, the vehicle tests whether $m$ has already been received. To achieve this, each vehicle maintains a list of message $I D s$. An incoming warning message $I D$ is inserted in the list if $m$ is received for the first time (i.e. its ID has not been previously stored in the list), and if so it is rebroadcasted to the surrounding vehicles only when the distance $d$ between sender and receiver is higher than a distance threshold $D$, or the receiver is in a different street than the sender. We consider that two vehicles are in a different street when: (i) both are indeed in different roads (this information is obtained by on-board GPS systems with integrated street maps), or (ii) the receiver, in spite of being in the same street, is near to an intersection. Hence, warnings can be rebroadcasted to vehicles which are traveling on other 


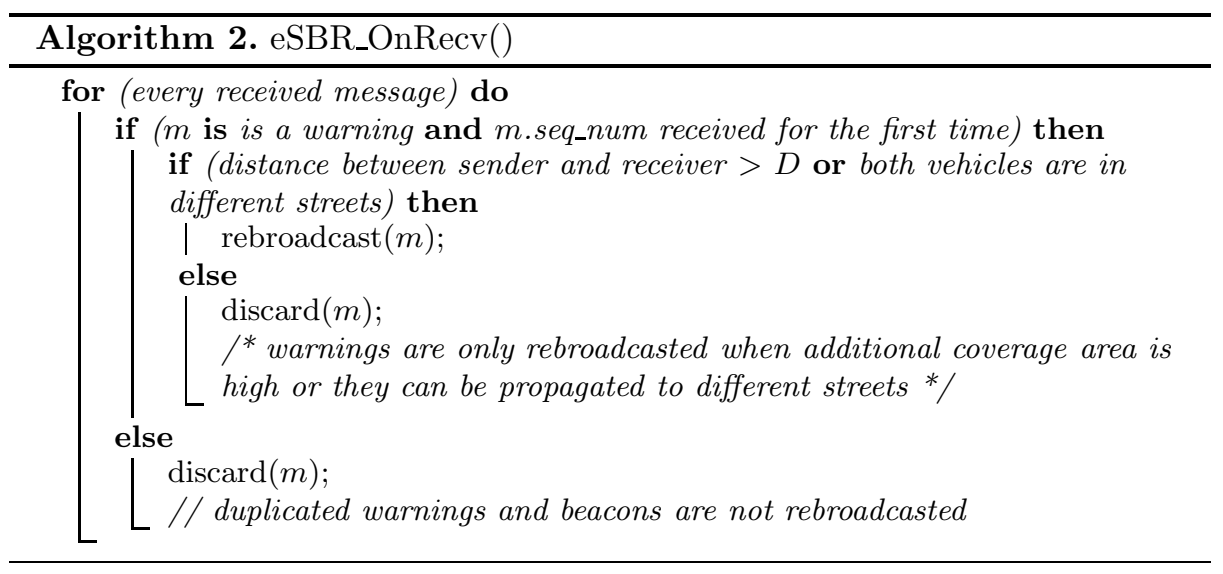

streets, overcoming the radio signal interference due to the presence of buildings. If the message is a beacon, it is simply discarded since we are not interested in the dissemination of beacons.

Figure 1 shows an example in a real map scenario where shaded polygons represent buildings. When vehicle $A$ broadcasts a warning message, it is only received by neighboring vehicles $B, C$, and $D$ because buildings interfere with the radio signal propagation. In this situation, if we use distance or location-based

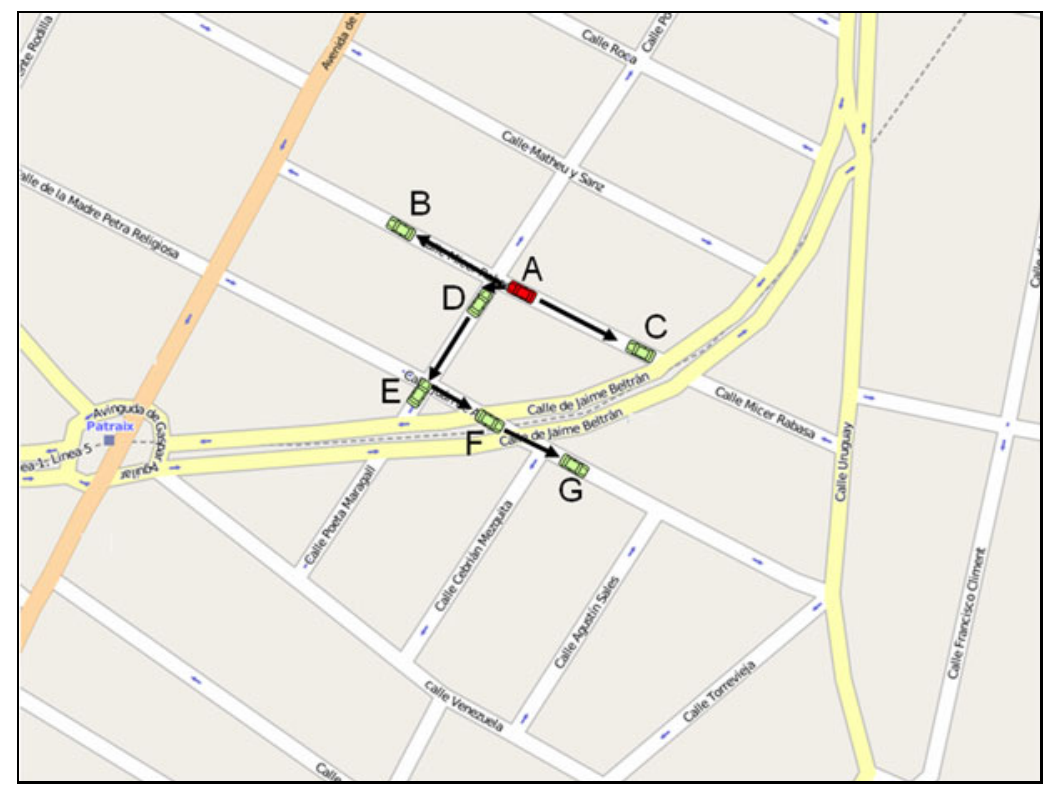

Fig. 1. The enhanced Street Broadcast Reduction scheme: example scenario taken from the city of Valencia in Spain 
schemes, vehicles $B, C$, and $D$ will rebroadcast the message only if distances $d 1, d 2$ and $d 3$, respectively, are large enough (i.e., the distance is larger than a distance threshold $D$ ), or its additional coverage areas are wide enough (i.e., the $\mathrm{AC}$ is larger than a coverage threshold $A$ ). So, supposing that only vehicle $C$ meets this condition, the warning message could still not be propagated to the rest of vehicles (i.e., $E, F$, and $G$ ).

Our eSBR scheme solves this problem as follows. In eSBR, vehicle $D$ will rebroadcast the warning message since vehicle $D$ is in a different street than vehicle $A$. In this way, the warning message will arrive to all the vehicles represented in only four hops. In modern Intelligent Transportation Systems (ITS), vehicles are equipped with on-board GPS systems containing integrated street maps. Hence, location and street information can be readily used by eSBR to facilitate dissemination of warning messages. When the additional coverage area is wide enough, vehicles will rebroadcast the received warning message. However, when the additional coverage area is very low, vehicles will rebroadcast warning messages only if they are in a different road.

Note that distance and location schemes can be very restrictive, especially when buildings interfere with radio signal propagation. Without eSBR, warning messages will not arrive at vehicles $E, F$ and $G$ due to the presence of buildings.

\section{Simulation Environment}

Deploying and testing VANETs involves high cost and intensive labor. Hence, simulation is a useful alternative prior to actual implementation. VANET simulations often involve large and heterogeneous scenarios. Compared to MANETs,

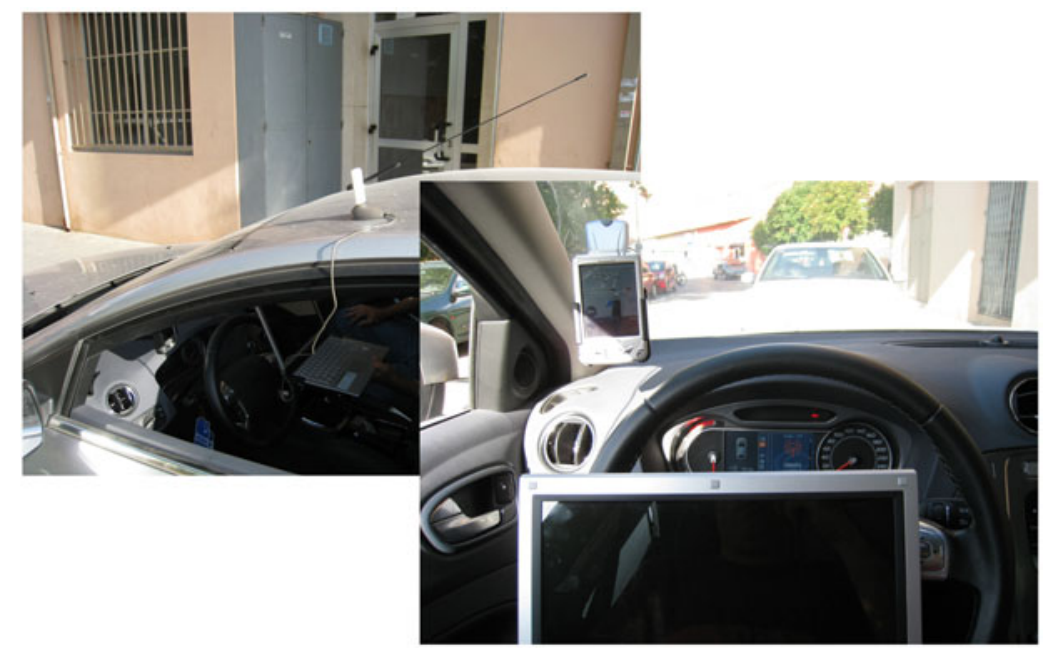

Fig. 2. Images of the experiment to determine the radio signal attenuation due to distance between vehicles 


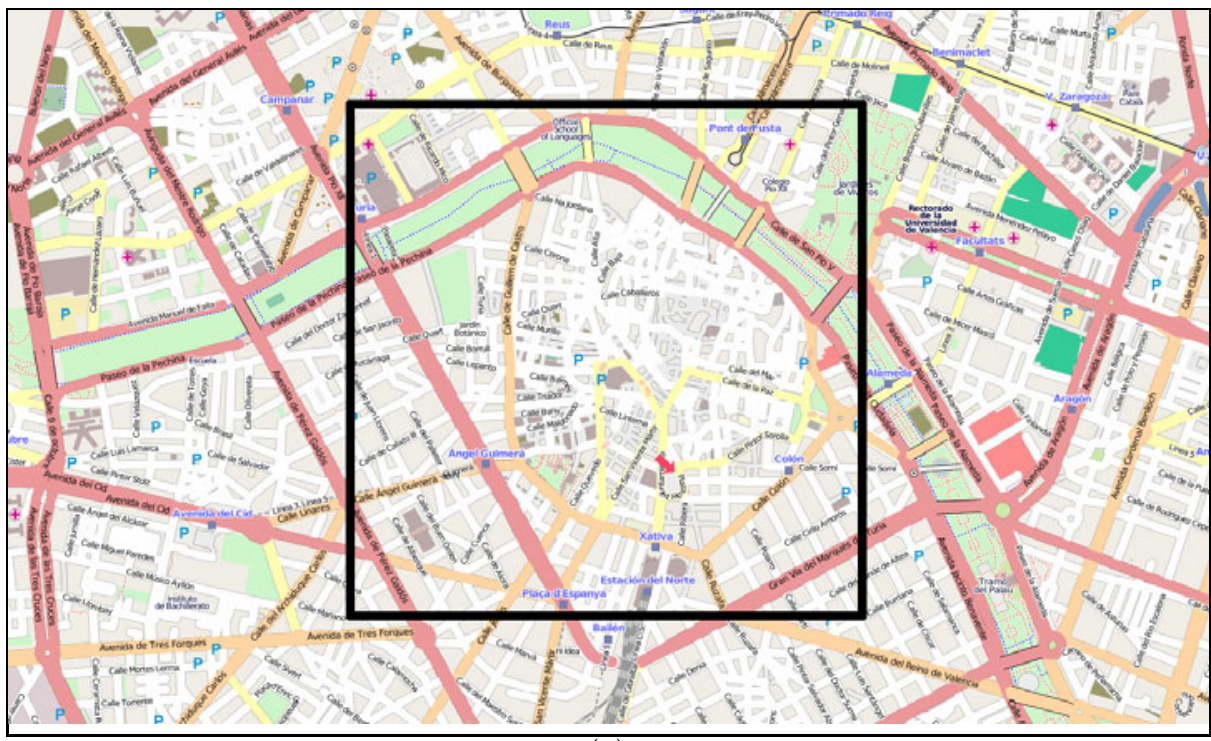

(a)

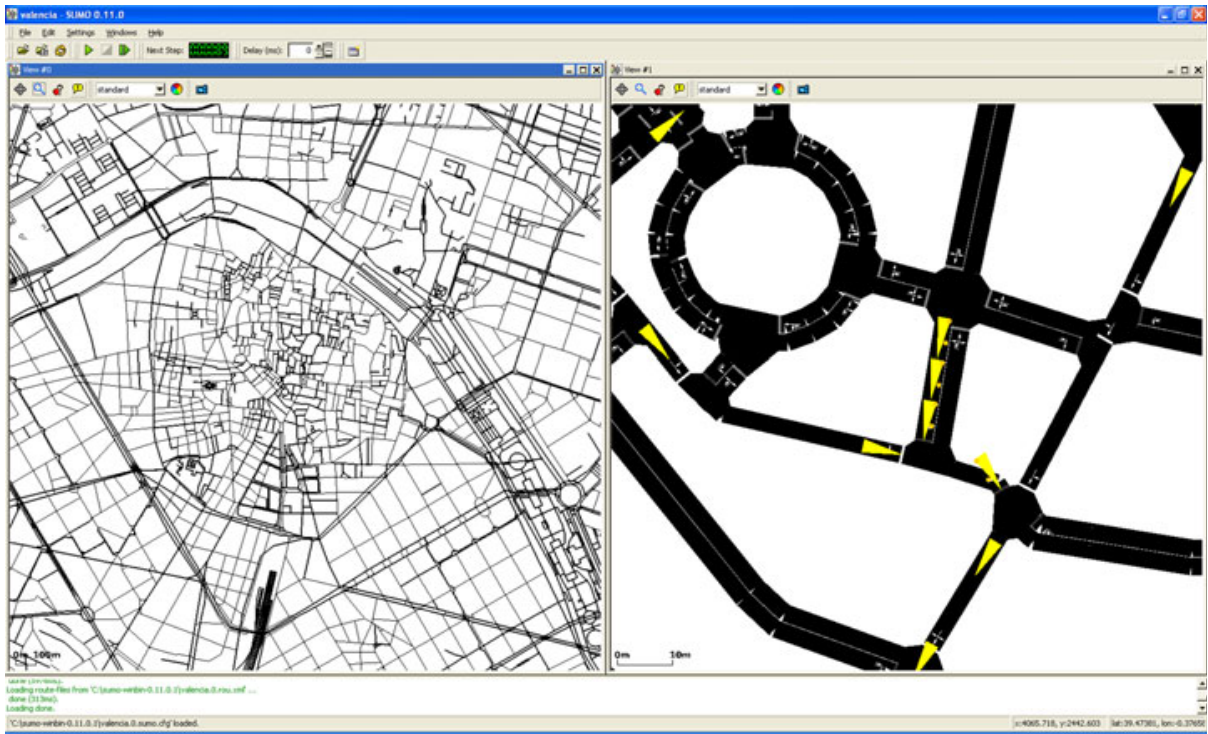

(b)

Fig. 3. Simulated scenario of Valencia city: (a) OpenStreetMap layout, and (b) the SUMO converted version. The box shows our simulated area. 
VANET simulations must account for some extra characteristics that are specific to vehicular environments [6].

In this section, we present our VANET simulation setup. Simulation results presented in this paper were obtained using the ns- 2 simulator. We modified the simulator to follow the upcoming Wireless Access in Vehicular Environments (WAVE) standard closely. Achieving this requires extending the ns-2 simulator to implement IEEE 802.11p, which is a draft amendment to the IEEE 802.11 standard that defines enhancements to support Intelligent Transportation Systems (ITS) applications.

In terms of the physical layer, the data rate used for packet broadcasting was fixed at $6 \mathrm{Mbit} / \mathrm{s}$, i.e., the maximum rate for broadcasting in 802.11p when assuming a $20 \mathrm{MHz}$ channel. The MAC layer is based on the IEEE 802.11e Enhanced Distributed Channel Access (EDCA) Quality of Service (QoS) extensions. Therefore, application messages are categorized into different Access Categories (ACs), where AC0 has the lowest, and AC3 the highest priority. The contention parameters used for the Control Channel $(\mathrm{CCH})$ are shown in [7]. Thus, in our warning message dissemination mechanism, warning messages have the highest priority (AC3) at the MAC layer, while beacons have lower priority (AC1). Moreover, since we are simulating real city maps with buildings, we have modified the ns-2 simulator to model the impact of distance and obstacles in signal propagation. The Radio propagation model used was the Real Building and Distance Attenuation Model (RBDAM) model, a new model we have implemented inside ns-2 which is based on the formerly proposed BDAM model [8]. RBDAM considers the signal attenuation due to the distance between vehicles based on real data obtained from experiments in the $5.9 \mathrm{GHz}$ frequency band using the IEEE 802.11a standard (see Figure 2), with a maximum transmission range of 400 meters, and also accounts for the presence of buildings (i.e., communication among vehicles is only possible when they are within line-of-sight).

To perform realistic simulations, it is specially important that the chosen mobility generator will be able to obtain a detailed microscopic traffic simulation and to import network topologies from real maps. Our mobility simulations are performed with SUMO 9], an open source traffic simulation package which has interesting microscopic traffic capabilities such as: collision free vehicle movement, multi-lane streets with lane changing, junction-based right-of-way rules, traffic lights, etc. (see Figure 3b). SUMO can also import maps directly from map databases such as OpenStreetMap [10] and TIGER [11].

The simulated topology represents the downtown area of Valencia city in Spain, and it was obtained from OpenStreetMap. Figure 3 shows the layout used in our simulations.

\section{Simulation Results}

In this section, we perform a detailed analysis to evaluate the impact of the proposed eSBR scheme on the overall system performance. We compare the impact of our scheme in two different scenarios: a Manhattan grid scenario, and 
a realistic city map, i. e., the city of Valencia in Spain. Since performance results are highly related to the specific scenarios used, and due to the random nature of the mobility model, we performed fifteen simulations to obtain reasonable confidence intervals. All the results shown here have a $90 \%$ confidence interval. Each simulation lasted for 450 seconds. In order to achieve a stable state before gathering data traffic, we only started to collect data after the first 60 seconds.

We evaluated the following performance metrics: (a) percentage of blind vehicles, (b) warning notification time, and (c) number of packets received per vehicle. The percentage of blind vehicles is the percentage of vehicles that do not receive the warning messages sent by accidented vehicles. These vehicles remain blind because of their positions, due to packet collisions, or due to signal propagation limitations. The warning notification time is the time required by normal vehicles to receive a warning message sent by a "warning mode" vehicle (a vehicle that broadcasts warning messages). Table11 shows the simulation parameters used.

In this paper, we evaluated the performance of our eSBR proposed scheme with respect to: (i) a distance-based scheme, and (ii) a location-based scheme, using two different scenarios: (i) a Manhattan grid-style, and (ii) a real map of Valencia, a city from Spain, using an average density of 75 vehicles $/ \mathrm{km}^{2}$. The impact of other parameters affecting VANET warning message dissemination, such as the density of vehicles, the priority and periodicity of messages, etc., was previously studied in 12 .

Table 1. Parameter values for the simulations

\begin{tabular}{|l|c|}
\hline \hline Parameter & Value \\
\hline number of vehicles & 300 \\
map area size & $2000 \mathrm{~m} \times 2000 \mathrm{~m}$ \\
maximum speed & $50 \mathrm{~km} / \mathrm{h}$ \\
distance between streets (in Manhattan) & $100 \mathrm{~m}$ \\
number of warning mode vehicles & 3 \\
warning packet size & $256 b y t e s$ \\
normal packet size & $512 b y t e s$ \\
packets sent by vehicles & 1 per second \\
warning message priority & AC3 \\
normal message priority & AC1 \\
MAC/PHY & $802.11 \mathrm{p}$ \\
Radio Propagation Model & $\mathrm{RBDAM}$ \\
maximum transmission range & $400 \mathrm{~m}$ \\
eSBR distance threshold (D) & $300 \mathrm{~m}$ \\
\hline \hline
\end{tabular}

Figure 4 shows the warning notification times obtained. When the simulation scenario is a Manhattan grid (see Figure 4a), the system needs only one second to inform at least $75 \%$ of the vehicles for all the schemes, and the message dissemination process ends in less than 2 seconds. The percentage of vehicles receiving the warning message is an $8 \%$ higher when using eSBR compared to both the distance-based and the location-based schemes. Concerning the 
Manhattan scenario, warning messages reach longer distances because of the lack of direction changes in the straight streets that characterize this map.

When simulating a real map scenario (see Figure 4b), some noticeable differences appear. First of all, the percentage of vehicles receiving warning messages decreases from about the $90-95 \%$ in a Manhattan scenario to less than $50 \%$, since the complexity of the layout and the arrangement of the buildings of real maps significantly interfere with the signal propagation process. Thus, the highly directional radio signal reduces drastically the probability of reaching longer distances. As for the warning notification time, we obtained better results using the eSBR scheme. The warning messages reach $40 \%$ of the vehicles in 0.45 seconds. Also notice that the message dissemination process ends before 1 second for all schemes. This time is $50 \%$ lower than the obtained in the Manhattan scenario because, in a real scenario, the information is only spread to nearby vehicles.

As for the percentage of blind nodes, Figure [5] shows how it will largely depend on the chosen simulation scenario. There are few blind vehicles when simulating a Manhattan scenario for all schemes (less than 15\%), and the percentage of blind nodes is reduced by half when using eSBR. Nevertheless, less than $50 \%$ of the vehicles are aware of warning messages when simulating a real city map. If we use this more realistic scenario we find that, when the eSBR scheme is adopted, the percentage of vehicles which receive warning messages increases by $8 \%$ when compared to the distance and location-based techniques.

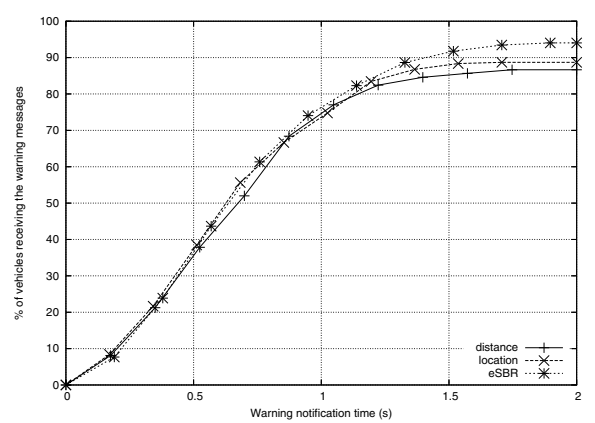

(a)

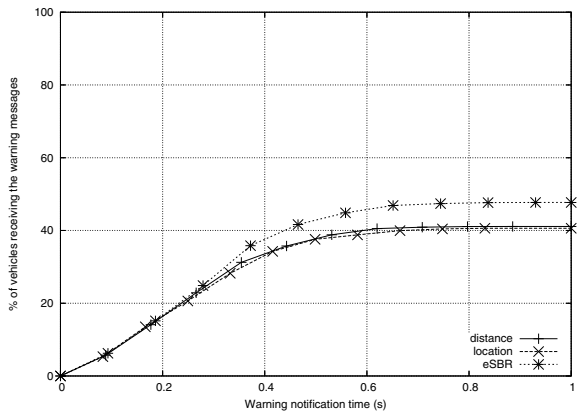

(b)

Fig. 4. Average propagation delay when varying the simulation scenario: (a) using a Manhattan layout, and (b) using a real map

Finally, Figure [5] shows the total number of packets received per vehicle, which is a measure of the degree of contention in the channel. In the Manhattan scenario the signal propagates easily due to the streets' position, and so the vehicles receive many duplicated messages. However, in a real map scenario, the more complex layout makes difficult signal propagation, and so the number of messages received decreases. These messages are received only by vehicles which are likely to face the dangerous situation, i.e. they are in the same street or in 


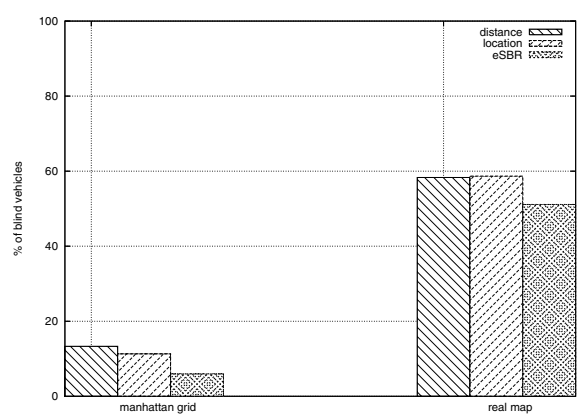

(a)

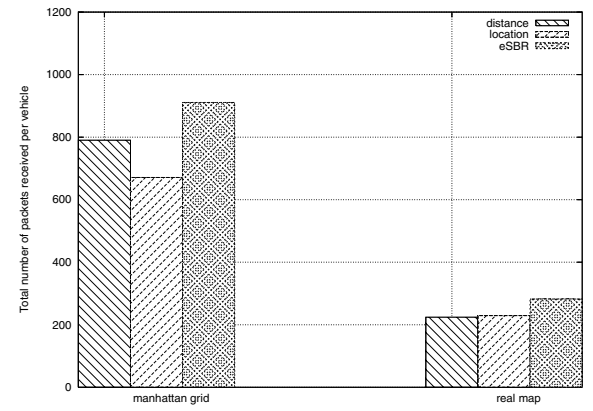

(b)

Fig. 5. (a) Percentage of blind vehicles vs. scenario layout, and (b) Total number of packets received per vehicle vs. scenario layout, both accounting for a Manhattan and a real map scenario

nearby ones. When simulating the real city map, the number of packets received per vehicle is almost the same for the distance and location-based schemes; when using eSBR the number of packets received increases slightly, but the percentage of vehicles which receive warning messages increases to a greater extent.

Authors in [1] demonstrated that the location-based scheme was more efficient than the distance-based scheme, since it reduces redundancy without compromising the number of vehicles receiving the warning message. Nevertheless, the main drawback for using this scheme is the high computational cost of calculating the additional coverage. Our simulation results demonstrate that eSBR outperforms both these schemes without introducing much complexity and calculations.

\section{Conclusion}

Achieving efficient dissemination of messages is of utmost importance in vehicular networks to warn drivers of critical road conditions. However, broadcasting of warning messages in VANETs can result in increased channel contention and packet collisions due to simultaneous message transmissions. In this paper, we introduce the enhanced Street Broadcast Reduction (eSBR) scheme to reduce broadcast storm in real map urban scenarios and to improve the performance of warning message dissemination. Simulation results show that eSBR outperforms other schemes in high density urban scenarios, yielding a lower percentage of blind vehicles while drastically alleviating the broadcast storm problem, being thus suitable for real scenarios. Our experiments also highlight that the message propagation behavior in realistic scenarios based on maps of actual cities differs greatly from more traditional Manhattan-style scenarios. Thus, we consider that the results obtained using unrealistic scenarios should be revised, and we recommend the adoption of real maps whenever possible. 


\section{Acknowledgments}

This work was partially supported by the Ministerio de Educación y Ciencia, Spain, under Grant TIN2008-06441-C02-01, and by the Fundación Antonio Gargallo, under Grant 2009/B001.

\section{References}

1. Tseng, Y.-C., Ni, S.-Y., Chen, Y.-S., Sheu, J.-P.: The broadcast storm problem in a mobile ad hoc network. Wireless Networks 8, 153-167 (2002)

2. Suriyapaibonwattana, K., Pomavalai, C.: An effective safety alert broadcast algorithm for VANET. In: International Symposium on Communications and Information Technologies (ISCIT), October 2008, pp. 247-250 (2008)

3. Suriyapaiboonwattana, K., Pornavalai, C., Chakraborty, G.: An adaptive alert message dissemination protocol for VANET to improve road safety. In: IEEE Intl. Conf. on Fuzzy Systems (FUZZ-IEEE), August 2009, pp. 1639-1644 (2009)

4. Korkmaz, G., Ekici, E., Ozguner, F., Ozguner, U.: Urban multi-hop broadcast protocols for inter-vehicle communication systems. In: Proceedings of First ACM Workshop on Vehicular Ad Hoc Networks (VANET 2004) (October 2004)

5. Wisitpongphan, N., Tonguz, O.K., Parikh, J.S., Mudalige, P., Bai, F., Sadekar, V.: Broadcast storm mitigation techniques in vehicular ad hoc networks. IEEE Wireless Communications 14, 84-94 (2007)

6. Martinez, F.J., Toh, C.-K., Cano, J.-C., Calafate, C.T., Manzoni, P.: A survey and comparative study of simulators for vehicular ad hoc networks (VANETs). Wireless Communications and Mobile Computing (October 2009)

7. Eichler, S.: Performance evaluation of the IEEE 802.11p WAVE communication standard. In: Proceedings of the Vehicular Technology Conference (VTC 2007 Fall), Baltimore, MD, USA (September 2007)

8. Martinez, F.J., Toh, C.-K., Cano, J.-C., Calafate, C.T., Manzoni, P.: Realistic Radio Propagation Models (RPMs) for VANET Simulations. In: IEEE Wireless Communications and Networking Conference (WCNC), Budapest, Hungary (2009)

9. SUMO, Simulation of Urban MObility (2009), http://sumo.sourceforge.net

10. OpenStreetMap, collaborative project to create a free editable map of the world (2009), http://www.openstreetmap.org

11. TIGER, Topologically Integrated Geographic Encoding and Referencing (2009), http://www. census.gov/geo/www/tiger

12. Martinez, F.J., Cano, J.-C., Calafate, C.T., Manzoni, P.: A Performance Evaluation of Warning Message Dissemination in 802.11p based VANETs. In: IEEE Local Computer Networks Conference (LCN), Switzerland (October 2009) 